\title{
La Accesibilidad Universal y el Diseño para Todos desde la perspectiva de género
}

\author{
Universal Accesibility and Design for All \\ from a gender perspective
}

\section{Resumen}

Ser mujer con discapacidad hoy supone un reto añadido si se aplica a los diferentes modelos de mujer que conviven en la realidad social. Aunque las políticas destinadas a este sector de mujeres deban ser específicas, una necesidad vital une a las mujeres con y sin discapacidad: desarrollarse como mujeres completas en un mundo en demasiada ocasiones hostil, donde todo tipo de servicios relacionados con las tecnologías de la información y de la comunicación (TIC), los entornos físicos, etc., se convierte en nada cuando se obvia la premisa principal, diseñar pensando en todos y en todas. Por ello presentamos esta reflexión sobre la situación actual y las actuaciones que son necesarias para posicionar a las mujeres con discapacidad en igualdad con el resto de la ciudadanía.

\section{Palabras clave}

Diseño para Todos, servicios públicos, género.

\begin{abstract}
Being a disabled woman today presents an added challenge when considered in light of the many different types of women of today's society. Although politics in this area ought to be specific, one vital necessity unites women with and without disabilities: to emerge as fulfilled women in a world that is too often hostile, where every type of service related to information technology and communication, physical environments, etc., becomes nothing when the principal premise is disregarded, to create designs that take every person into account. For this reason, we present this reflection about the current situation and the actions required to achieve equal opportunities for disabled women.
\end{abstract}

\section{Keywords}

Design for All, public services, gender.

\section{Yolanda $\mathbf{M}^{\mathrm{a}}$ de la Fuente Robles \\ <ymfuente@ujaen.es> \\ Coordinadora del Máster en Accesibilidad Universal y Diseño para Todos \\ Vicerrectora de Igualdad, Cultura y Cooperación al Desarrollo de la Universidad Internacional de Andalucía \\ Facultad de Trabajo Social. \\ Universidad de Jaén-España}

\section{Jesús Hernández-Galán}

Director de Accesibilidad Universal Fundación ONCE

Coordinador del Máster en

Accesibilidad Universal y Diseño para Todos. Universidad de Jaén-España
Para citar:

De La Fuente Robles, Y. y HernándezGalán, J. (2OI4): "La Accesibilidad Universal y el Diseño para todos desde la perspectiva de género". Revista Española de Discapacidad, 2 (I): I I 5-I 29.

$<$ http://dx.doi.org/I0.5569/23405104.02.01.06>

Fecha de recepción: I4-OI-2OI4 Fecha de aceptación: 29-04-20I4 


\section{Introducción}

Las mujeres han salido del entorno privado, han sido capaces de interiorizar las políticas que han ido dirigidas a ellas para dotarlas de libertad e igualdad, pero el entorno todavía no ha sido capaz de dar respuesta a todos los cambios que iban encaminados a la búsqueda de la accesibilidad en igualdad. Ha llegado el momento de que las mujeres con discapacidad sean visibilizadas en todas las facetas de la vida y en los diferentes roles que como cualquier persona pueda realizar: como niña, con posibilidad de acceso al juego en entornos comunes (parques, colegios, etc.); como adolescente, viviendo su etapa de desarrollo en situación de normalidad (cines, polideportivos, piscinas, espacios de ocio, etc.); como mujer, viviendo su sexualidad, con un acceso igualitario a consultas ginecológicas diseñadas para todo tipo de mujeres, a su maternidad y posterior cuidado de sus descendientes, y cómo no, a vivir su vejez de manera activa.

Asimismo, al analizar la discapacidad desde la perspectiva de género se puede observar cómo los distintos estudios sobre el tema (Campling,I98I; Lonsdale,I990; Morris,I996; García, 2004; Moya, 2004; Soler, Teixeira y Jaime 2008), tienden a ubicar la discapacidad en un contexto social, en el que hay que desplegar todos los recursos necesarios para que la mujer con discapacidad salga de su "invisibilidad", que se conozca su realidad para poder sortear las dificultades que le impiden el pleno acceso a la vida laboral, cultural, social, política, educativa, etc., en igualdad de condiciones que el resto de población.

Por otro lado, desde un punto de vista normativo, nos encontramos ante una escasa conjugación entre el binomio génerodiscapacidad y, consecuentemente, ante una insuficiente información estadística al respecto que permita conocer la realidad de las mujeres y niñas en situación de discapacidad así como las formas de discriminación que les afectan. Ahora bien, la incorporación de la perspectiva de género en las políticas de discapacidad no supone abogar exclusivamente por la no discriminación en cuestión de discapacidad y género, sino que conlleva un efectivo avance en las políticas de igualdad para todas las personas, de modo que, a través de éstas se logre terminar con las desigualdades estructurales y, en definitiva, alcanzar una amplia cohesión social.

A tenor de lo expuesto, las mujeres con discapacidad experimentan una realidad con muchos más obstáculos que los hombres con discapacidad, según datos del Comité Español de Representantes de Personas con Discapacidad (CERMI), lo que da lugar a situaciones diversas de discriminación y dificultades específicas (CERMI, 2002).

El CERMI elaboró a través de su Área de Género, el "II Plan Integral de Acción de Mujeres con Discapacidad 2013-2016", como herramienta estratégica para impulsar sistemáticamente las políticas públicas de género y discapacidad.

La situación de discriminación y exclusión de las mujeres y niñas con discapacidad, así como la de las mujeres cuidadoras de personas con discapacidad, sigue siendo en nuestro país un problema de primer orden que exige la adopción de medidas específicas que incidan sobre las causas reales que le dan origen.

El Plan ha servido para mostrar cómo la confluencia de dos ejes de discriminación, como el género y la discapacidad, generan situaciones de violación de derechos humanos que han pasado totalmente inadvertidas y que merecen ser abordadas de manera sistemática a partir de un nuevo enfoque, que permita poner el acento en aquellos ámbitos donde las organizaciones de personas con discapacidad y del movimiento de mujeres no se han detenido. Un plan de estas características se enmarca en las denominadas “medidas de acción positivas”, entendidas como estrategias que tienen un carácter limitado en el tiempo y que cuyo objetivo es contribuir a eliminar prejuicios, actitudes, discursos y prácticas que dificultan a un determinado grupo social alcanzar una situación real de igualdad de oportunidades; en este caso, las mujeres y niñas 
Cuadro 1. Dificultades específicas de la mujer con discapacidad

\begin{tabular}{|c|c|}
\hline Dificultad & Consecuencia \\
\hline $\begin{array}{l}\text { Sobreprotección } \\
\text { familiar }\end{array}$ & $\begin{array}{l}\text { Dificultades que impiden el ejercicio de una vida independiente, así como el } \\
\text { establecimiento de relaciones sociales. Consecuentemente, un gran número de } \\
\text { mujeres con discapacidad se ven relegadas a una situación de aislamiento social. De } \\
\text { este modo, desarrollan un nivel de autonomía y toma de decisiones menor que los } \\
\text { hombres con discapacidad en particular y el resto de población en general. }\end{array}$ \\
\hline Barreras & $\begin{array}{l}\text { Diferencias en función del tipo de discapacidad. Así, a las mujeres con discapacidad } \\
\text { sensorial les afectan más las barreras de acceso a la información, y a las mujeres con } \\
\text { discapacidad física las barreras arquitectónicas, con la consecuente inaccesibilidad } \\
\text { a diversos servicios y recursos (centros comerciales, transporte, consultas } \\
\text { ginecológicas, casas de acogida, etc.). }\end{array}$ \\
\hline Acceso al empleo & $\begin{array}{l}\text { Implica dependencia económica, bien a través pensiones no contributivas, cuya } \\
\text { cuantía no alcanza el salario mínimo interprofesional, y/o de la administración de sus } \\
\text { propios ingresos por parte de la familia, lo que perpetúa su situación de invisibilidad } \\
\text { y dependencia. }\end{array}$ \\
\hline $\begin{array}{l}\text { Acceso a la } \\
\text { formación }\end{array}$ & $\begin{array}{l}\text { La mujer con discapacidad presenta mayor dificultad a la hora de acceder a la } \\
\text { formación, en relación con los hombres con discapacidad y por supuesto con la } \\
\text { población en general. Esto deriva en una elevada tasa de analfabetismo funcional o } \\
\text { total entre ellas. La percepción social como incapaces conlleva una nula estimulación } \\
\text { para participar en los procesos formativos y de capacitación. }\end{array}$ \\
\hline Imagen corporal & $\begin{array}{l}\text { Sobre todo en mujeres con discapacidad física manifiesta, el valor que se le da en } \\
\text { la sociedad a la imagen corporal provoca en ellas un proceso de negación de la } \\
\text { sexualidad así como una disminución de su autoestima ya que su imagen no se } \\
\text { corresponde con los cánones de belleza establecidos socialmente. }\end{array}$ \\
\hline $\begin{array}{l}\text { Relaciones } \\
\text { sociales }\end{array}$ & $\begin{array}{l}\text { La discapacidad tiene una influencia negativa el desarrollo de las relaciones sociales, } \\
\text { acentuada en la relación de pareja. } \\
\text { Esta concepción generalizada deriva en una disminución de su autoestima, lo que se } \\
\text { traduce en una reducción de las posibilidades de acceso a distintos ámbitos sociales } \\
\text { y laborales. }\end{array}$ \\
\hline
\end{tabular}

Fuente: Martín, 2013.

con discapacidad fundamentalmente, aunque no en exclusiva ${ }^{\mathrm{I}}$.

En definitiva, como podemos observar, la percepción social respecto a la mujer con discapacidad (asexuada, poco capacitada intelectual, laboral y relacionalmente, etc.), enfatiza la discriminación, cuanto menos doble (por su condición de mujer y por su condición de persona con discapacidad), sufrida por las mujeres, jóvenes y niñas con discapacidad.

I. Información complementaria en II Plan de Acción de Mujeres con Discapacidad 20I3-20I6: <http://www.cermi.es/ES-ES/ NOTICIAS/Paginas/Inicio.aspx? TSMEIdNot=4644>.
Por ello, no podemos dejar de lado la inclusión del gender mainstreaming o transversalización de género, como estrategia para promover la igualdad de género, adoptada de manera explícita en el ámbito de la cooperación internacional en la Plataforma para la Acción de Beijing en I995, la misma se refiere a la necesidad de influir en todas las metodologías, análisis, políticas y planificación desde una perspectiva de género: “(...) los gobiernos y otros actores deben promover una política activa y visible de integración de la perspectiva de género en todas las políticas y programas y, para ello, antes de que se adopten las decisiones, debe hacerse un análisis de los efectos sobre las mujeres y los hombres, respectivamente". 
La transversalización de género se refiere al hecho de que las cuestiones de igualdad de género se integren en todas las políticas públicas, dejando de ser cuestiones marginales o asociadas solamente a determinadas políticas sociales tradicionales (como familia, por ejemplo) y proponiéndose como una estrategia complementaria a las "políticas nacionales de la mujer" o "políticas específicas dirigidas a las mujeres". Éstas enfocan directamente determinados problemas de la desigualdad de género, pero que no son suficientes para contrarrestar los efectos de desigualdad que puedan tener las políticas generales.

Y este es el caso de las políticas de discapacidad, ya que el uso del género en su diseño se hace imprescindible para conseguir implicar en el desarrollo de instituciones y políticas más afines a los derechos humanos, al desarrollo humano, a la participación, a la paridad y, en definitiva, promotoras de cohesión social. El acceso, con igualdad de oportunidades y sin discriminación, a activos y servicios públicos de calidad que permitan a la ciudadanía el ejercicio de sus derechos fundamentales, ya que en una dimensión que pone acento en las personas, la cohesión social implica la construcción de ciudadanía y de sentimientos de pertenencia, sobre todo a través de la participación activa de distintos colectivos en la construcción de un espacio público que facilite la búsqueda de objetivos comunes (López, 2007: 5-2I).

Ya que si todo lo señalado se obvia, da lugar a entornos discapacitantes y exclusógenos si algunos de sus factores de uso cotidiano lo son, lo que constituye un grave problema sobre el que se hace necesario incidir. La importancia de adecuar los productos y servicios a las características reales de los hombres y las mujeres puede ser, en muchas ocasiones, suficiente para favorecer su vida independiente. No obstante, la aplicación de los principios igualitarios focalizados en la construcción de espacios universalmente accesible para hombres y mujeres, por un lado, y la integración de las personas con falta de autonomía personal, por otro, olvida a menudo que los espacios vitales de hombres y mujeres aún no han alcanzado una equivalencia real, por lo que se aplican principios integradores, como la accesibilidad universal, a la población en general, soslayando la parte por el todo.

\section{Las reglas del juego en la accesibilidad conocidas y olvidadas}

La Convención de los Derechos de las Personas con Discapacidad (2006), en su artículo 9 relativo a la accesibilidad, señala que: "para que las personas con discapacidad puedan vivir de forma independiente y participar plenamente en todos los aspectos de la vida, los Estados Partes adoptarán las medidas apropiadas para garantizar a las personas con discapacidad el acceso, en igualdad de condiciones con los demás, al entorno físico, el transporte, la información y las comunicaciones, incluidos los sistemas y las tecnologías de la información y las comunicaciones, así como otras instalaciones y servicios públicos o abiertos al público, tanto en zonas urbanas y en las zonas rurales áreas. Estas medidas, que incluirán la identificación y la eliminación de obstáculos y barreras de acceso, se aplicarán, entre otras cosas:

a. Edificios, carreteras, transporte y náuticos, lagos y instalaciones al aire libre, como escuelas, viviendas, instalaciones médicas y los lugares de trabajo;

b. Información, comunicaciones y otros servicios, incluidos los servicios electrónicos y de emergencia.

Los Estados Partes también adoptarán las medidas apropiadas para:

a. Desarrollar, promulgar y supervisar la aplicación de normas mínimas y directrices sobre la accesibilidad de instalaciones y servicios públicos o abiertos al público;

b. Asegurar que las entidades privadas que proporcionan instalaciones y servicios públicos o abiertos al público tengan en cuenta todos los aspectos de la accesibilidad para personas con discapacidad; 
c. Proporcionar capacitación a los interesados en la accesibilidad sobre los problemas que enfrentan las personas con discapacidad;

d. Dotar a los edificios y otras instalaciones abiertas al público de señalización en Braille y en formatos fáciles de leer y comprender;

e. Ofrecer formas de asistencia humana o animal e intermediarios, incluyendo guías, lectores y profesionales de la lengua de signos intérpretes, para facilitar el acceso a edificios y otras instalaciones abiertas al público;

f. Promover otras formas adecuadas de asistencia y apoyo a personas con discapacidad para asegurar su acceso a información;

g. Promover el acceso de personas con discapacidad a las nuevas tecnologías de información y las comunicaciones y los sistemas, incluida Internet;

h. Promover el diseño, desarrollo, producción y distribución de la información y las comunicaciones accesibles a tecnologías y sistemas en una etapa temprana, de modo que estos sistemas y tecnologías sean accesibles al menor costo".

Igualmente recoge la Estrategia Europea sobre Discapacidad 2010-2020 la existencia de un compromiso renovado para una Europa sin barreras, ya que la accesibilidad se entiende como el acceso de las personas con discapacidad, en las mismas condiciones que el resto de la población, al entorno físico, al transporte, a las TIC y a otras instalaciones y servicios. La Estrategia señala que todavía hay barreras importantes en todos estos ámbitos, por ello se hace necesario garantizar la accesibilidad a los bienes y servicios, en especial los servicios públicos y los dispositivos de apoyo para las personas con discapacidad.

Asimismo, pone en marcha un proceso destinado a capacitar a las personas con discapacidad de manera que puedan participar plenamente en la sociedad, en unas condiciones de igualdad con el resto de la población. Dado el envejecimiento de la población de la Unión Europea (UE), estas medidas repercutirán visiblemente en la calidad de vida de una proporción creciente de la ciudadanía europea, exhortando a las instituciones de la UE y a los Estados miembros a colaborar en el marco de esta Estrategia con el fin de construir una Europa para todos libre de barreras ${ }^{2}$.

El acceso no es un acto o un estado, sino que más bien se refiere a la libertad de elección en cuanto a la forma de intervenir, abordar, informar o hacer uso de una situación. El entorno puede ser el conjunto en general o parte de él o la situación a la que se accede. La participación en condiciones de igualdad sería una realidad si se garantizara la igualdad de oportunidades para participar a través de medidas que mejoren la accesibilidad. Los elementos de la accesibilidad son atributos de la disponibilidad del entorno pero no son características del entorno.

La investigación en el campo de la rehabilitación de las personas con discapacidad ha identificado cinco características del entorno:

I. Accesibilidad - ¿puede llegar a dónde quiere ir?

2. Alojamiento - ¿puede hacer lo que quiere hacer?

3. Disponibilidad de recursos - ¿se cubren sus necesidades especiales?

4. Apoyo social - ¿le aceptan las personas que le rodean?

5. Igualdad - ¿recibe un trato igual al de los demás?

Estas características no proporcionan una base sobre la que clasificar los entornos, sino que más bien representan un esquema para clasificar las diferentes formas en las que las personas interactúan con los entornos; la accesibilidad es sólo una de las cinco características de los entornos accesibles identificados.

El diseño universal también establece una base para valorar la accesibilidad con referencia a las interacciones entre las personas y el entorno. Ya

2. Comunicación de la Comisión al Parlamento Europeo, al Consejo, al Comité Económico y Social Europeo y al Comité de las Regiones. Estrategia Europea sobre Discapacidad 2010-2020: Un Compromiso Renovado para una Europa sin Barreras. 
que la propuesta de valores del diseño universal es el diseño de productos y entornos que puedan ser usados por todas las personas (hombres y mujeres con o sin discapacidad), en la mayor medida posible.

Las dimensiones universales de acceso deberían: reconocer el contexto social, considerar la situación de la persona, tener en cuenta la edad y los factores culturales y apoyar los análisis en lo que se refiere a la persona y el entorno.

Aprovechando la Clasificación de Discapacidades desarrollada por la Organización Mundial de la Salud, los participantes en una reunión consultiva de expertos celebrada en Toronto (2002) en colaboración con las Naciones Unidas, establecieron las siguientes dimensiones para valorar el acceso:

I. Orientación, ¿quién? - ¿tiene la información que desea?

2. Independencia, ¿qué? - ¿elige lo que quiere hacer?

3. Movilidad, ¿dónde? - ¿va a dónde desea?

4. Empleo del tiempo, ¿cuándo? - ¿se compromete cuando quiere?

5. Integración social, ¿con quién? - ¿le aceptan los demás?

6. Autosuficiencia económica, ¿con qué? ¿tiene los recursos que necesita?

7. Transición, cambio - ¿está preparado para el cambio?

Una de las consecuencias de las evaluaciones del entorno, basadas en las dimensiones universales relativas a las interacciones entre las personas y sus entornos, es que éstas permiten un examen y evaluación de las variables de la accesibilidad y la identificación de opciones para reducir la exclusión social y garantizar así los derechos para todos. La accesibilidad no es un asunto que interese sólo a un grupo social específico, sino que es un requisito indispensable para el progreso de todos ${ }^{3}$.

3 Información complementaria en "La ONU y las personas con discapacidad": <http://www.un.org/spanish/esa/social/ disabled/disacc.htm>.
La falta de accesibilidad al entorno y a los servicios afecta de una manera muy específica a las mujeres, no solo a las mujeres con discapacidad, sino que como bien sabemos, gran parte de las personas que se dedican a cuidar a personas mayores, o personas con algún tipo de dependencia, son mujeres. El entramado urbanístico, el diseño de los edificios, las redes de transportes o la configuración de las telecomunicaciones hacen aún más difícil la vida de muchas mujeres, tradicionalmente confinadas al espacio doméstico y que, sin embargo, se ven obligadas a hacer uso de un espacio público muchas veces no pensado para ellas.

\section{Entorno físicos y virtuales predefinidos que invalidan y excluyen}

La configuración de los espacios públicos ha sido diseñada tradicionalmente, desde la perspectiva de la "estandarización", un punto de vista que considera la existencia de un patrón medio de persona, excluyendo de este patrón a las personas desde el punto de vista de las personas con discapacidad. De este modo, se ha ido constituyendo un entorno discriminante que no contempla la diversidad, el género y se olvida del concepto de calidad de vida. El 40 por ciento de la ciudadanía española, se ve afectada por estos obstáculos según datos del Plan Nacional de Accesibilidad 2004-20I 2.

Superado el citado Plan, y una vez aprobado el Real Decreto Legislativo I/2013, de 29 de noviembre, por el que se aprueba el Texto Refundido de la Ley General de derechos de las personas con discapacidad y de su inclusión social, según señala la Disposición adicional cuarta dedicada a los "Planes y programas de accesibilidad y para la no discriminación: El Gobierno, en el plazo de un año desde la entrada en vigor de esta ley, aprobará un plan nacional de accesibilidad para un periodo de nueve años. El plan se desarrollará a través de fases de actuación trienal. En su diseño, aplicación y seguimiento 
participarán las asociaciones de utilidad pública más representativas en el ámbito estatal de las personas con discapacidad y sus familias".

Todo esto lleva aparejado una nueva planificación estratégica a largo plazo que se hace necesaria sin mayor dilación, debido a que pese a que discapacidad y accesibilidad se han relacionado íntimamente de forma tradicional, las barreras a la movilidad y a la comunicación afectan a todos los miembros de una sociedad que aumenta su esperanza de vida. Desde el imaginario social se percibe este problema como una traba que afecta por igual a personas con discapacidad sean del sexo que sean; sin embargo, las desigualdades en materia de accesibilidad continúan coartando sus derechos. Así, por ejemplo, en España son muchas más las mujeres que hacen uso del transporte público, y en esto las mujeres con discapacidad no son una excepción. La falta de recursos económicos hace más complicado a las mujeres contar con un vehículo propio adaptado. Por eso la falta de accesibilidad del transporte público incide de una manera muy negativa en las mujeres del colectivo.

Desde el punto de vista del diseño, es muy importante que en el mundo de arquitectura y el urbanismo se tienda poco a poco a diseños mucho más cuidados del espacio público, ya que la irrupción de la mujer en el mundo del diseño arquitectónico está ayudando a proyectar desde un punto de vista diferente, mucho más conciliador e integrador, que genera espacios de inclusión dentro de la ciudad ${ }^{4}$.

Analizar la participación de la mujer en los principios de la accesibilidad universal y transformar sus normas de aplicabilidad supondría transformar los productos y entornos en elementos activos de intervención encaminados a promover la autonomía personal, el mantenimiento y mejora de la salud y, en definitiva, la mejora de la calidad de vida de las mujeres.

4. Información complementaria en: <http://laciudadaccesible. ideal.es/20I I/o3/accesibilidad-y-mujer/>.
Por tanto, el objetivo principal debe ser el identificar la representación de la mujer en los principios rectores de la accesibilidad universal y las formas en las que están representados sus espacios sociales en los productos de apoyo a la autonomía personal.

La idea es terminar con los diseños basados en la división machista del uso del espacio y tiempo, a su vez amplificado cuando hablamos de personas con discapacidad. De manera tradicional el hombre ha ocupado la esfera pública, urbana y social, y la mujer la privada, doméstica, etc. Al romper con esta premisa se considera fundamental acercarnos a un nuevo diseño que dé respuesta a la verdadera realidad social, alejada de roles ya superados, mucho más amplia y compleja.

Hay que trabajar en el fomento de la autodeterminación que lleve a dar el paso desde el concepto de demandante de atención y de cuidados, al de ciudadanía plena, construyendo una sociedad democrática e integradora y no excluyente de personas que no puedan desarrollar sus derechos básicos como consecuencia de su discapacidad ${ }^{5}$.

\section{El entorno físico diseñado para todos y} todas

El concepto de entorno accesible implica que se debe ampliar el término "estándar", utilizado en el diseño en general, usando el concepto de manera que dentro del mismo tengan cabida la diversidad de personas que habitan o utilizan un determinado entorno.

Sin embargo, las normas uniformes sobre la igualdad de oportunidades para las personas con discapacidad, aprobadas por la Asamblea

5. Conclusiones del I Congreso Internacional sobre Mujer y Discapacidad, más información: <http://centreantigona. uab.cat/docs/articulos/I\% 20 Congrés $\%$ 2ointernacional $\% 20$ sobre $\%$ 2oDona $\%$ 20i\% 2odiscapacitat. \% 20Conclusions.pdf $>$. 
General de las Naciones Unidas mediante Resolución 48/96 de 20 de diciembre de I993, en su apartado II, en las esferas previstas para la igualdad de participación, no hacen referencia a la inclusión de la perspectiva de género, aunque sí señalan que "Los Estados deben reconocer la importancia global de las posibilidades de acceso dentro del proceso de lograr la igualdad de oportunidades en todas las esferas de la sociedad. Para las personas con discapacidades de cualquier índole, los Estados deben: a) establecer programas de acción para que el entorno físico sea accesible, y b) adoptar medidas para garantizar el acceso a la información y a la comunicación" (Art 5. Posibilidades de acceso).

De igual modo, el Plan Estatal de Accesibilidad (I999), en su introducción recogía que "la accesibilidad integral implica accesibilidad en la edificación, urbanismo, transporte, comunicación y servicios. Es un derecho básico que garantiza la no discriminación por razones de edad, discapacidad o características funcionales y es condición previa para la participación social y económica en igualdad de oportunidades”. El sexo, siendo determinante en el fenómeno social y demográfico de la discapacidad, no se especifica, por lo que esto supone una invisibilización de los espacios de las mujeres en el diseño de la edificación, el urbanismo, el transporte, la comunicación y los servicios y la consiguiente inclusión de la perspectiva de género en el desarrollo legislativo, técnico, económico, de mercado, planificación, diseño, ejecución, rehabilitación, formación y mentalización. El proceso de empoderamiento de la mujer que se está dando en todo los aspectos de la vida se tiene que reflejar también en todo el sistema. No existe una ciudadanía tipo para el que diseñar el espacio público sino una ciudadanía plural a la que consultar y con la que crear el espacio colectivo.

Para lograr la accesibilidad integral se hace necesario analizar si el espacio y los servicios están pensados para generar vida, convivencia y seguridad; para ello hay que perseguir que la accesibilidad sea mucho más que la eliminación de barreras arquitectónicas, que corresponda a una realidad. En esta línea habría que apuntar los factores que tradicionalmente han señalado las mujeres como importantes:

- Reconocer el entorno fácilmente, saber dónde te encuentras y dónde está el lugar al que te diriges (fácil orientación y reconocimiento de los diferentes elementos del espacio).

- Ver y ser vistas.

- Oír y ser oídas.

- Tener siempre la visión de una salida o un lugar donde pedir ayuda.

- Limpieza y cuidado del entorno.

- Actuar de manera colectiva en el espacio público ${ }^{6}$.

\section{El entorno virtual eAccesible}

En el año 2008, una publicación del Instituto de la Mujer, "Mujeres y nuevas tecnologías de la información y la comunicación”, señalaba que las personas con discapacidad forman otro colectivo que por su prevalencia entre la población total debe ser tenido en cuenta en el análisis de la relación con las TIC de forma específica. Muy a menudo, el acceso de estas mujeres a las nuevas tecnologías se produce de forma desigual respecto al conjunto de la sociedad, generando a veces barreras difíciles de superar que requieren ser adaptadas. Con el tratamiento apropiado, fundamentalmente haciéndolas accesibles, las TIC pueden posibilitar que esas barreras se conviertan en elementos integradores y de cohesión social.

En esta línea se trabaja desde la iniciativa E-europe, que tiene, entre otros objetivos, uno relativo a la inclusión de las personas con

6. Estándares Generales para definir la seguridad de los espacios públicos, Bilbao, 2010: <http://www20.gencat.cat/docs/ interior/Home/OI0\% 20El\% 20 Departament/Programa \% 20 per $\%$ 20a $\%$ 2ol'equitat $\%$ 2ode $\%$ 2ogènere/Jornada $\%$ 20La $\%$ 20 seguretat $\%$ 2oals $\%$ 2opobles $\%$ 20i $\%$ 20a \% 2oles $\%$ 2ociutats $\%$ 2oamb \% 2ovisio \% 2ode \% 2ogenere/Documents/3a \% 20 IdoiaUriarte.pdf>. 
discapacidad en la economía del conocimiento; así, la iniciativa propone prestar especial atención a las personas con discapacidad en materia de inclusión digital para evitar la infoexclusión y para asegurar su plena participación en la Sociedad de la Información.

Una serie de factores influyen en la actitud hacia las TIC y en su correlativo nivel de uso. Como señala el documento "Discapacidad eAccesibilidad", dentro de los componentes socioculturales destaca, en primer lugar, el nivel formativo, seguido por la edad y el nivel económico; cuanto mayor es el nivel de estudios y la renta, mayor utilización de la tecnología.

Además de los anteriores, hay una serie de causas que influyen de manera considerable, como es el grado de dependencia y/o de autonomía de la persona y el grado de accesibilidad del dispositivo tecnológico en cuestión: cuanto mayor es el nivel de accesibilidad mayor es la predisposición hacia las TIC. Debemos aprender de los errores del pasado. La actividad realizada para eliminar las barreras con las que se creó el medio físico (arquitectura, urbanismo, transporte, etc.) ha demostrado que es realmente costoso, tanto desde el punto de vista económico como temporal, hacer accesible algo inicialmente no diseñado para todos, “a posteriori”. En el ámbito tecnológico, todavía se está a tiempo de evitar una situación similar a la descrita. Las TIC deberían ser herramientas al servicio de las personas; sin embargo, el abanico de necesidades y diversidad de individuos que componen una sociedad es bastante más amplio de lo que los diseñadores de productos y servicios tienen en mente.

El estado de la tecnología actual dispone de medios suficientes y elementos conocidos para hacer accesible los desarrollos tecnológicos a prácticamente cualquier persona, con independencia de sus aptitudes. Hoy las personas con discapacidad cuentan con ayudas técnicas que les permiten desarrollar una vida plena y superar muchas de las barreras, invisibles para los demás, que se encuentran a diario. Mejorar la integración de este colectivo es un reto y una oportunidad. Si el objetivo se consigue, las TIC traerán consigo innumerables beneficios, ventajas y nuevas oportunidades de trabajo, de formación, de ocio, y, en definitiva, de normalización (Miranda, 2007).

En un lugar destacado de la accesibilidad universal, se sitúa la participación de la mujer en los espacios virtuales, una cuestión a resolver para alcanzar los supuestos igualitarios de los que parte el concepto de diseño para todos. Por ello, desde diversos foros, se ha reconocido la importancia de que la mujer participe en las nuevas tecnologías, como en la Global Knowledge 97, I Conferencia Internacional que analizaba el papel de las tecnologías de la información y su impacto en los países en desarrollo. Esta tuvo una considerable participación femenina y constituyó un paso importante en la inclusión de las mujeres en la agenda del conocimiento para el desarrollo (Cabrera, 2005). En esta reunión, se constató la importancia de las TIC como un medio para reunir y distribuir los conocimientos de las mujeres y planteó que todas las facetas de ingeniería, diseño, desarrollo y entrega de las TIC deben incluir la igualdad de género si no se quiere perpetuar la desigualdad como en otros ámbitos de participación de la vida social. Siendo, por ello, acciones prioritarias el incorporar el análisis de género a toda la investigación de política científica y tecnológica, desarrollando y subvencionando evaluaciones del impacto de las nuevas TIC en las necesidades de comunicación de las mujeres, y proporcionando adiestramientos, sistemas de acceso y entrega, incluyendo versiones desarrolladas para mujeres.

En el año 2003, las conclusiones convenidas de la Comisión de la Condición Jurídica y Social de la Mujer ${ }^{7}$ sobre la participación y el acceso de la mujer a los medios de difusión y las tecnologías de la información y las comunicaciones y sus repercusiones en el adelanto y la potenciación del papel de la mujer, así como su utilización a tal efecto, fueron las siguientes ${ }^{8}$ :

7. Aprobadas por la Comisión de la Condición Jurídica y Social de la Mujer en su $47^{\circ}$ periodo de sesiones 8. Informe completo en: <http://www.un.org/womenwatch/>. 
“Apoyándose en los objetivos estratégicos y las medidas establecidas en la Declaración y Plataforma de Acción de Beijing'y el documento final aprobado en el vigésimo tercer período extraordinario de sesiones de la Asamblea General titulado "Igualdad entre los géneros, desarrollo y paz para el siglo XXI" ${ }^{\circ}$, sobre la contribución de los medios de difusión y de las tecnologías de la información y las comunicaciones al adelanto y la potenciación del papel de la mujer y en la Declaración del Milenio ${ }^{\text {II }}$ y sus Objetivos de Desarrollo para promover la igualdad entre los sexos y la autonomía de la mujer como métodos eficaces de combatir la pobreza, el hambre y las enfermedades, de estimular un desarrollo verdaderamente sostenible; y de garantizar que todos tengan acceso a los beneficios de las nuevas tecnologías, en especial en las esferas de la información y las comunicaciones, esta Comisión observa que existen notables diferencias a nivel mundial en cuanto a la participación de la mujer en las Tecnologías de la información y la comunicación; sobre todo en lo que respecta a su contenido, producción, el acceso a ellos y su utilización”.

Igualmente, señala la importancia de observar desde una perspectiva de género estas nuevas tecnologías al objeto de frenar la brecha digital que actualmente se está asentando en nuestras sociedades.

En sentido contrario, estos instrumentos, puestos al servicio de la ciudadanía, pueden potenciar la igualdad de géneros en diferentes dimensiones: (i) para facilitar el acceso de la mujer al conocimiento y a la toma de decisiones, (ii) para ofrecer nuevas oportunidades de comunicación adaptada a la población femenina y a la población femenina con problemas de movilidad y carencias en la autonomía personal.

Además, la Comisión considera necesario, en primer lugar, dar prioridad a la inclusión de

9. Informe de la Cuarta Conferencia Mundial sobre la Mujer, Beijing, 4 a I 5 de septiembre de 1995 .

Io. Resolución S-23/3 de la Asamblea General.

I I. Resolución 55/2 de la Asamblea General. la perspectiva de género en cualquier política de corte nacional o de otro ámbito territorial así como en la legislación, los proyectos y los instrumentos técnicos reguladores y crear mecanismos de supervisión y rendición de cuentas para que se apliquen las normas y reglamentos relacionados con las cuestiones de género, en colaboración con mujeres especialistas en tecnología de la información, organizaciones de mujeres y agentes sociales defensores de la igualdad; y en segundo lugar, considera necesario promover la participación de la mujer en el control y la gestión de las tecnologías de la información y las comunicaciones y los medios de difusión. También recomienda reforzar la capacidad de los mecanismos para el adelanto de la mujer, mediante la asignación de recursos suficientes y adecuados y la aportación de conocimientos especializados, para asumir un papel de vanguardia en la promoción de la igualdad de género en los medios de difusión y las tecnologías de la información, y apoyar la participación de esos mecanismos en los procesos nacionales, regionales e internacionales relacionados con los medios de difusión y las tecnologías de la información y las comunicaciones.

Por otro lado, la Unión Internacional de Telecomunicaciones (UIT), a raíz de la resolución adoptada en I998 en la Conferencia Mundial para el Desarrollo de las Telecomunicaciones, creó el Grupo de Expertos sobre los Temas de Género (TFGI). El objetivo de este grupo era asegurar que los beneficios de las telecomunicaciones y de las nuevas tecnologías emergentes no discriminaran a las mujeres y estas estuvieran incluidas en los procesos generados de contratación, empleo, formación y participación, pero prueba de que este objetivo no se ha cumplido es la situación en la que nos encontramos actualmente, donde se hace necesario añadir al mismo los conceptos de accesibilidad y género.

La División de las Naciones Unidas para el Progreso de la Mujer efectuó en Corea, en el año 2002, una Reunión del Grupo de Expertos sobre "tecnologías de la información y las 
comunicaciones y su impacto y empleo como instrumento para el desarrollo y la habilitación de las mujeres". Enfatizaron la necesidad de incluir la perspectiva de género en las nuevas tecnologías y pusieron sobre la mesa diferentes prácticas realizadas por mujeres discapacitadas y excluidas para integrarse y capacitarse. Ello sirvió como punto de partida para la sesión de la Comisión de la Condición de la Mujer de 2003, así como para la primera fase de la "Cumbre Mundial de la Sociedad de la Información”. La Declaración de Principios y Plan de Acción de esta cumbre sintetiza las demandas de los grupos de mujeres y organizaciones internacionales a través de las siguientes recomendaciones adoptadas:

- "Habilitación para una participación plena”. La alfabetización y la educación primaria universal son factores esenciales para crear una Sociedad de la Información plenamente integradora, teniendo en cuenta en particular las necesidades especiales de las niñas y las mujeres (Declaración de principios, Creación de Capacidades, párrafo 4).

- "Procurar eliminar los obstáculos de género que dificultan la educación y la formación en materia de TIC, y promover la igualdad de oportunidades de capacitación para las mujeres y niñas en los ámbitos relacionados con las TIC". Se debe incluir a las niñas entre los programas de iniciación temprana en las ciencias y la tecnología, para aumentar el número de mujeres en las carreras relacionadas con las TIC. Promover el intercambio de prácticas óptimas en la integración de los temas de género en la enseñanza de las TIC (Plan de Acción, Desarrollo de Capacidades, párrafo I Ig).

- "Se alienta a los gobiernos a que, en colaboración con las partes interesadas, definan políticas de las TIC que propicien la actividad empresarial, la innovación y la inversión, haciendo especial hincapié en la promoción de la participación de la mujer" (Plan de Acción, Ambiente propicio, párrafo I3).

- “Alentar la definición de prácticas óptimas para los cibertrabajadores y los ciberempleadores basadas, a escala nacional, en los principios de justicia e igualdad de género y en el respeto de todas las normas internacionales pertinentes" (Plan de Acción, Ciberempleo, párrafo I9a).

En definitiva, el tema del acceso es fundamental en el discurso de género y las TIC. El acceso está indisolublemente relacionado con la disponibilidad de la infraestructura necesaria y en casi todos los países en desarrollo las infraestructuras de comunicación son menos estables y están menos disponibles en las áreas rurales y urbanas pobres, donde vive la mayoría de las mujeres. Como señalaron el Fondo de Desarrollo de las Naciones Unidas para la Mujer (UNIFEM) y la United Nations University/ Technical College (UNU/TECH): “A las mujeres, que tienen responsabilidades especiales con los niños y los ancianos, les resulta más difícil que a los hombres migrar a los pueblos y ciudades. Por consiguiente, el carácter urbano de la posibilidad de conexión priva a las mujeres, más que a los hombres, del derecho universal a la comunicación" (Ambrosi, Peugot y Pimienta, 2005).

En cuanto a las nuevas tecnologías como integradoras de personas en situación de dependencia, estas siempre han desempeñado un papel fundamental para el desarrollo de la autonomía de las personas con discapacidad, en lo que respecta a la ayuda a la comunicación, a la movilidad, informática, domótica o innovaciones que permiten orientar sus desplazamientos con la tecnología Global Positioning System (GPS). Estas tecnologías se han desplegado progresivamente alrededor de dos ejes: el desarrollo de soluciones tecnológicas, por una parte, la información, la sensibilización y el lobbying político (Roy, 2005).

Por ello, las mujeres discapacitadas deben poder acceder a las nuevas tecnologías y ejercer sus derechos como ciberciudadanas. Ejemplo de ello son las normativas que Estados Unidos, Australia, Reino Unido y Francia, que han desarrollado el derecho de accesibilidad a los sitios web públicos. Otro ejemplo es la iniciativa de la web World Wide Web Consortium (WC3) 
que ha creado la de Iniciativa de Accesibilidad Web, conocida como Web Accessibility Initiative (WAI). Se trata de una actividad cuyo objetivo es facilitar el acceso de las personas con discapacidad, desarrollando pautas de accesibilidad, mejorando las herramientas para la evaluación y reparación de accesibilidad Web, llevando a cabo una labor educativa y de concienciación en relación a la importancia del diseño accesible de páginas Web, y abriendo nuevos campos en accesibilidad a través de la investigación en este área ${ }^{12}$.

\section{Conclusiones}

En definitiva, la doble discriminación del colectivo de mujeres con discapacidad es un hecho que se ha visibilizado en los últimos años, al evidenciarse una discriminación de las mujeres con discapacidad incluso en las acciones positivas dirigidas al colectivo de personas en situación de dependencia.

En cambio, si hacemos referencia al espacio virtual, la mujer accede desde la igualdad que le proporciona el conocimiento, en función del acceso igualitario a la educación de los tiempos presentes, pero desde el sesgo que genera su menor participación en los espacios de saber de las tecnologías. No obstante, una vez en la red, su trayectoria de búsqueda y navegación le lleva a acceder a los espacios sociales virtuales de una forma paralela a la forma en que lo hace en los espacios urbanos. La mujer se adueña de los contenidos que reproducen sus intereses en la vida cotidiana.

La conquista de espacios y posiciones sociales por parte de la mujer en las últimas décadas es un hecho suficientemente apreciable en el momento actual, constatado por la evidencia del acceso a la educación, al trabajo y a las instituciones de participación democrática. El coste de este proceso es alto y genera además un escenario

I2. Información complementaria en: <http://www.w3c.es/>. en el cual existe una segregación ocupacional del mercado de trabajo y diferentes formas de discriminación para las mujeres, donde aparece otro reto generado por los nuevos ámbitos procedentes de la revolución de la sociedad informacional, en particular por la comunidad virtual. Las nuevas infraestructuras precisas para el acceso a estas redes y las condiciones necesarias para el disfrute de las mismas generan a su vez nuevas fragmentaciones. Otra vez, el acceso al conocimiento se presenta como una complejidad añadida al ya proceloso camino marcado por las polaridades de las diferentes realidades sociales a nivel mundial.

Esta reflexión nos lleva a plantearnos varios interrogantes: ¿es posible el acceso a los nuevos ámbitos de la estructura social y, en particular, de mercado, sin que se generen ciertos caos en aquellos espacios tradicionalmente asumidos por la mujer?, ¿es la incorporación de la mujer al conocimiento, en igualdad de condiciones a los hombres, una realidad en proceso? o, en sentido contrario, ¿suponen los cambios sociales de los últimos años el surgimiento de nuevos escenarios de participación acompañados de crecientes fragmentaciones que impiden la anexión en condiciones igualitarias? Por ende, la participación en los nuevos espacios dibujados por la sociedad informacional es un condicionante imprescindible para el reparto equitativo del poder. La cuestión es si los nuevos modelos basados en principios de accesibilidad generan nuevas exclusiones o si no son más que las consecuencias de su implantación en la misma sociedad fragmentada (Sotomayor, 2007).

En definitiva, el concepto de accesibilidad de género o accesibilidad universal desde la perspectiva de género afecta a dos ámbitos fundamentales de la vida social: el acceso a la información y a la tecnología y el acceso a los espacios urbanos. No obstante, ambos transitan hacia un mismo campo de accesibilidad: lo social, lo público, la información, la libertad, el conocimiento, la sabiduría. Pudiéramos por lo tanto fundir el concepto de accesibilidad telemática y accesibilidad urbana a través de la sinergia generada por la perspectiva de género, en un único espacio accesible para hombres y 
mujeres sin barreras físicas ni virtuales (De la Fuente y Sotomayor, 2010).

Debemos consensuar mujeres y hombres un nuevo modelo de sociedad y ciudadanía, vertebrado alrededor de una redefinición de lo público y lo privado, introduciendo una diferente racionalidad de los tiempos. Es imprescindible que los medios se impliquen en la transformación necesaria de los tópicos que responden al interés decidido de que las cosas continúen como están en cuanto a la imagen de la mujer y de los papeles que hombres y mujeres están llamados a desempeñar (Chicano, 2002).

Asimismo, respecto a las mujeres en situación de dependencia, si bien se observa una evolución positiva en cuanto al acceso a la educación, al igual que el resto de mujeres, ésta no se corresponde con una mayor posibilidad de acceso al mercado laboral. Del mismo modo, cabe desatacar las iniciativas, públicas y privadas, que en los últimos años se han venido desarrollado desde un enfoque de género como herramienta de cambio y mejora respecto a las mujeres con discapacidad, aunque, de momento, los resultados sean más bien moderados.

En definitiva, aún persisten en nuestra sociedad grandes sesgos de género, a través de los cuales se determinan funciones en función del sexo tanto a nivel público como privado, lo que conlleva unos efectos negativos en el colectivo de mujeres en general pero sobre todo en las mujeres con discapacidad en particular, ya que éstas encuentran mucha más discriminación y restricción de oportunidades. Obviamente el riesgo de exclusión es mucho mayor en las mujeres, no solo en relación con los hombres sino también respecto a las mujeres en general, ya que la violencia y el aislamiento están más presentes en ellas que en cualquier otro grupo social.

Por tanto, en vistas a un futuro próximo se hace cada vez más necesario adoptar medidas que eliminen los roles femeninos y fomenten el acceso de las mujeres con discapacidad a los programas y servicios de autonomía personal, aumentar la participación de las mujeres en los cargos directivos de administraciones, organizaciones, empresas y otras entidades de prestación de apoyos a personas con discapacidad, extender trasversalmente la dimensión de discapacidad en las actuaciones encaminadas a reducir la desigualdad de género e incorporar la perspectiva de género en todos los estudios, análisis estadísticos, epidemiológicos y planes de actuación orientados a la población con discapacidad (Huete, 20II).

Como señala ONU Mujeres (20I3) en el Documento "Un objetivo transformador e independiente para lograr la igualdad de género, los derechos y el empoderamiento de las mujeres: Imperativos y Componentes Claves", no podemos obviar que un objetivo transformativo e independiente tiene que estar fundamentado en la comprensión de que las causas estructurales de la desigualdad basada en género se hallan en sistemas de discriminación que a menudo se justifican en el nombre de la cultura, la historia o la identidad de grupo, así como en la racionalización de las políticas que supone que la mejor forma de lograr la igualdad de género es reducir el papel del Estado y liberar "el mercado".

Para abordar estos sistemas de discriminación y la institucionalización de la subordinación de las mujeres que estos sistemas crean, el objetivo tiene que ser entre otros el poner fin a las historias de sub-inversión en el aumento de las capacidades y los recursos de mujeres y niñas e invertir la marginación sistemática de las mujeres en la toma de decisiones públicas y privadas. No termina en la paridad de género en el acceso a bienes y recursos, sino que busca promover una transformación en las relaciones de género que avanzarían la libertad de todos y todas. Visto que el nuevo marco sin duda determinará la naturaleza del desarrollo durante mucho tiempo, el mundo no puede correr el riesgo de perder esta oportunidad, que se presenta solamente una vez en una generación, para transformar las vidas de mujeres y niñas y los hombres y niños, en todas partes. 


\section{Referencias bibliográficas}

Cabrera Balleza, M. (2005): “Mujeres”. En Ambrosi, A.; Peugeot, V. y Pimineta, D. (coords.): Palabras en Juego: Enfoques Multiculturales sobre las Sociedades de la Información, Paris: C \& F Éditions (en línea). $<$ http://www.casanas.com.ar/artsAdj/Palabras_ en_juego-22I.pdf $>$, acceso 9 de octubre de 2012.

Cabrera, P. J. (2005): Nuevas Tecnologías y exclusión social. Un estudio sobre las posibilidades de las TIC en la lucha por la inclusión social en España, Madrid: Universidad Pontificia de Comillas, Fundación Telefónica.

Campling, J. (198I): Images of ourselves: women with disabilities talking, West Yorkshire: University of Leeds - Routledge and Kegan Paul Ltd.

Catherine, R. (2005): “Accesibilidad". En Ambrosi, A.; Peugeot, V. y Pimineta, D. (coords.): Palabras en Juego: Enfoques Multiculturales sobre las Sociedades de la Información, París: C \& F Éditions (en línea). <http://www.casanas. com.ar/artsAdj/Palabras_en_juego-22I.pdf>, acceso 9 de octubre de $20 \mathrm{I} 2$.

CERMI (I 999): Plan Estatal de Accesibilidad del CERMI. Madrid: Comité Español de Representantes de Personas con Discapacidad (en línea). <http://www.infomedula.org/ documentos/planaccesibilidadcermi.pdf $>$, acceso I 8 de septiembre de 20 I 2 .

CERMI (2002): Seminario Mujer con Discapacidad: múltiples dimensiones (en línea). <http://antiguo.cermi.es/graficos/especiales/ mdmd2002.asp>, acceso 27 de diciembre de 2012.

Comisión Europea (2009): Estrategia Europea sobre Discapacidad 2010-2020 (en línea). $<$ http://eurlex.europa.eu/LexUriServ/ LexUriServ.do?uri=COM:20I0:0636:FIN:ES:P $\mathrm{DF}>$, acceso 5 de agosto de $20 \mathrm{I} 2$.

Chicano, E. (2002): "Feminismo y Discapacidad". En Domínguez, C. (coord.): Mujer y
Discapacidad: un análisis pendiente, Oviedo: Principado de Asturias, Consejería de Asuntos Sociales: 39-70.

De la Fuente Robles, Y. M. y Sotomayor Morales, E. M. (20IO): “Accesibilidad Universal y Diseño para todas y todos”. En Crespo Garrido, M. y Moretón Sanz, F. (dirs.): Discriminación por razón de edad y sexo. Retos pendientes del Estado Social, Madrid: Colex: I65-I90.

España. Real Decreto Legislativo I/20I3, de 29 de noviembre, por el que se aprueba el Texto Refundido de la Ley General de derechos de las personas con discapacidad y de su inclusión social. Boletín Oficial del Estado, num. 289 , de 3 de diciembre de 2013 , p. 95635 a 95673 .

García de la Cruz, J. J. (2004): El espejo social de la mujer con gran discapacidad: barreras sociales para retornar a una vida normal, Madrid: Fundamentos.

Huete, A. (20I I): "Mujeres con discapacidad. Igualdad de oportunidades desde una perspectiva de género". Revista Observatorio Estatal de Discapacidad, 3: 60-73.

IMSERSO (2003): Plan Nacional de Accesibilidad 2004-20I2. Madrid: Ministerio de Trabajo y Asuntos Sociales, IMSERSO (en línea). $<$ http://usuarios.discapnet.es/disweb200o/lex/ AccePlan2004-20I 2.pdf>, acceso 2 de julio de $20 \mathrm{I} 2$.

Lonsdale, S. (I990): Women and Disability: The Experience of Physical Disability among Women, Hampshire: Macmillan Education.

López, I. (2007): Género y Politicas de Cohesión Social, Madrid: Fundación Internacional y para Iberoamérica de Administración y Políticas.

Martín, $\mathrm{M}^{\mathrm{a}}$ C. (20I3): La transversalidad de género en las políticas públicas. Su aplicación en la Ley 39/2006 de I4 de diciembre de promoción de la Autonomía Personal y Atención a las Personas en Situación de 
Dependencia (en línea). <http://ruja.ujaen.es/ bitstream/ı0953/460/ı/9788484397267.pdf>, acceso 6 de enero de 2014 .

Miranda de Larra, R. (2007): Discapacidad eAccesibilidad, Madrid: Fundación Orange.

Morris, J. (1996): Encuentros con desconocidas. Feminismo y discapacidad, Madrid: Narcea.

Moya Maya, A. (2004): Mujer y Discapacidad: una doble discriminación, Huelva: Hergue Editora Andaluza.

Naciones Unidas (1995): Declaración de la Plataforma para la Acción de Beijing. La Cuarta Conferencia Mundial de la Mujer (en línea). <http://www.un.org/womenwatch/daw/ beijing/pdf/BDPfA \% 2oS.pdf $>$, acceso 8 de julio de 20 I 2.

Naciones Unidas (2006): Convención de los Derechos de las Personas con Discapacidad (en línea). <http://www.un.org/esa/socdev/ enable/documents/tccconvs.pdf $>$, acceso 4 de agosto de $20 \mathrm{I} 2$.

Naciones Unidas (2013): Un objetivo transformador e independiente para lograr la igualdad de género, los derechos y el empoderamiento de las mujeres: imperativos y componentes claves (en línea).
$<$ http://www.unwomen.org/es/what-we-do/ /me $\mathrm{dia} /{ }_{1} \mathrm{BCo}_{5} \mathrm{D}_{2} \mathrm{~A}_{7} \mathrm{~F}_{7} \mathrm{~A}_{4} \mathrm{EF}_{49}{ }_{9} \mathrm{E} 88 \mathrm{~F}_{9} \mathrm{FF}_{5} \mathrm{BE}_{7002}$. ashx>, acceso I4 de abril $20{ }_{4} 4$.

Naciones Unidas. Resolución 48/96, de 20 de diciembre de I993, Normas Uniformes sobre la igualdad de oportunidades para las personas con discapacidad (en línea). <http://www. un.org/spanish/disabilities/default.asp? $\mathrm{id}=498>$, acceso 8 de mayo de 2014 .

Organización Mundial de la Salud (2002): Clasificación de las Discapacidades. Naciones Unidas (en línea). <http://www.un.org/spanish/ esa/social/disabled/disacc.htm>, acceso I 6 de septiembre de $20 \mathrm{I} 2$.

Red2Red Consultores (2008): Mujeres y nuevas Tecnologías de la Información y Comunicación. Madrid: Ministerio de Igualdad, Instituto de la Mujer (en línea) <http://es.scribd.com/ doc/16472998/Mujeres-y-Nuevas-Tecnologiasde-la-Informacion-y-Comunicacion>, acceso I 6 de septiembre de $20 \mathrm{I} 2$.

Sotomayor Morales, E. (2007): "La exclusión social ante la globalización: el capital humano femenino". En Fernández Pantoja, P. y Cruz Blanca, M. J. (coords.): Igualdad de oportunidades y conciliación: una visión multidisciplinar, Barcelona: Editorial Bosch: I 5 5-I 78 . 